\title{
APPLICATION OF SLIME MOULD ALGORITHM FOR OPTIMAL ALLOCATION OF DATACOM AND PV SYSTEM IN REAL EGYPTIAN RADIAL NETWORK
}

\author{
Amal M. Abd El Hamed ${ }^{\mathrm{a}}$, Mohamed Ebeed ${ }^{\mathrm{b} *}$, Ahmed Refai ${ }^{\mathrm{b}}$, Montaser Abd El \\ Sattar ${ }^{\mathrm{c}}$, Adel A. Elbaset ${ }^{\mathrm{d}, \mathrm{e}}$, Taiea. A. Ahmed ${ }^{\mathrm{f}}$ \\ ${ }^{a}$ Electricity Department, Faculty of Technology and Education, Sohag University, Sohag, 82524, Egypt. \\ ${ }^{b}$ Department of Electrical Engineering, Faculty of Engineering, Sohag University, Sohag 82524, Egypt \\ ${ }^{c}$ Department of Electrical Engineering, Faculty of Engineering, South Valley University, Qena 83523, Egypt. \\ ${ }^{d}$ Electrical Engineering Department, Faculty of Engineering, Minia University, El-Minia, Egypt. \\ ${ }^{e}$ EL-Arish High Institute of Engineering and Technology, EL-Arish, North Sinai, Egypt. \\ ${ }^{f}$ Electrical Engineer in Qena Paper Company, Ques, Qena, Egypt.
}

\begin{abstract}
The aim of this paper to assess the optimal site and size of photovoltaic (PV) generation-based DG along with Distribution Static Synchronous Compensator (DSTATCOM) in the real distribution network East Delta Network (EDN). In this paper, a new optimization method called Slime Mold Algorithm (SMA) simulates the oscillation mode of a slime mold in nature. DSTATCOMs and PV modules are applied to reduce losses, voltage profile and enhance stability while meeting the limitations of equality and inequality in the system. Evaluation is provided with only PV modules installed, DSTATCOMs installed only and PV modules installed with DSTATCOM. The simulations verified that the optimization of PV modules combined with DSTATCOMs can significantly enhance system performance compared to PV only modules or DSTATCOMs and the effectiveness of the proposed algorithm for allocating PVs and DSTATCOMs in terms of objective functions.
\end{abstract}

(C) 2021 Published by Faculty of Engineering - Sohag University. DOI: 10.21608/SEJ.2021.155557.

Keywords: PV; DSTATCOM; Slime Mould Algorithm; Power losses; Real Distribution system.

\section{INTRODUCTION}

Integration of renewable distributed generators (RDGs) has more attention for researchers, and decision makers in electrical sectors for many technical, economic, environmental aspects as well is to cover the increasing load growth [1-3]. To maximize utilization of RDGs such as solar wind turbines, PV (photovoltaic), Hydro, and biomass etc. Numerous researchers focus on the techniques for enhancement of current network and its topology $[4,5]$ It worth mentioning that distribution networks (RDNs) has a high $\mathrm{R} / \mathrm{X}$ ratio compared with transmission system. Thus, RDNs suffer from poor power quality [6-8]. Inclusion the reactive power compensators paly important to solve the poor power quality problem of the RDNs. The reactive power compensator includes several distributed Flexible AC transmission systems (DFACTS) such as DSTATCOM, unified power quality conditioner and, Distribution Static Var Compensator (D-SVC),etc. [9-12].

DSTATCOM is a powerful device that is included in RDNs to control bus voltage. Moreover, DSTSTCOM has been integrated into RDNs for many tasks such as mitigating harmonics, reducing loss, improving stability, power factor correction, and reducing voltage deviations. [13]. All these tasks have been accomplished due to

${ }^{*}$ Corresponding author: mebeed@eng.sohag.edu.eg 
the DSTATCOM ability for injecting and absorbing reactive power to system very fast, dynamical responses made its application very wide within the field [14]. A comprehensive review has been presented in [15-17] to indicate the applications of the D-STATCOM in electrical system for performance enhancement.

Nowadays, the PV based DGs have been widely embedded in RDNs compared to the other renewable resources to supply the system of the required power instead of the conventional sources $[18,19]$. The integration of PV based DGs in electrical networks can provide peak shaving, reduce overloading of transmission lines, and reduce the greenhouse emissions. However, inclusion the PV system in an improper locations will affect the power quality of the RDNs [20,21]. Several efforts have been introduced for optimal inclusion the PV based DGs where the authors in applied for inclusion the PV and DSATCOM using Modified Ant Lion Optimizer under uncertainties of load and solar radiation [22]. An improved ecosystem algorithm has been implemented for optimal sizing PV, wind turbine (WI) and fuel cell [23]. Equilibrium optimizer (EO) has been employed for optimal size and site of the PV and WT in a Micro-grid [24]. The authors in [25]applied the Pathfinder Algorithm (PFA) to assign the PV units in multi-lateral RDNs. DSTATCOM is an efficient controller and it plays a significant role for improving the system performance. Several efforts have been presented for optimal sizing and placement the DSTATCOM in RDNs such as immune algorithm [26], Particle Swarm Optimization algorithm [27], JAYA optimization [28], bat algorithm [29], harmony search algorithm [30], improved sine cosine algorism [31] and grey wolf optimizer [32]

SMA is an efficient algorithm that simulates the behavior and morphological changes of a slime mold. Meanwhile, the use of weights in SMA is to simulate the positive and negative reactions produced by slime mold during foraging, thus forming three different types of morphs. [33]. In this paper, SMA is used to find the best locations and volumes of PV and DSTATCOMs in the true Egyptian distribution network. The paper's organization is listed as follows: Section 2 illustrates the formulation of the problem. Section 2 describes the steps for the SMA technique. Section 3 explains the simulation results. Section 4 depicts the final conclusions.

\section{Problem Formulation}

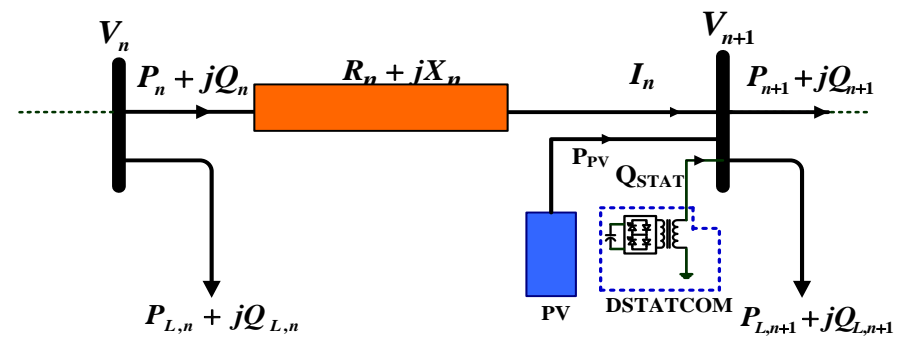

Fig.1. A simple RDNs with units and DSTATCOMs.

The RDNs consist of a set of branches, balanced power nodes, and constant loads. Equations (1) and (2) are used to describe the power flow solution with the integration of DSTATCOM and PV modules and are illustrated in Fig.1.

$$
\begin{aligned}
& P_{n+1}=P_{n}-P_{L, n+1}-R_{n, n+1}\left(\frac{P_{n}^{2}+j Q_{n}^{2}}{\left|V_{n}\right|^{2}}\right)+P_{P V} \\
& Q_{n+1}=Q_{n}-Q_{L, n+1}-X_{n, n+1}\left(\frac{P_{n}^{2}+j Q_{n}^{2}}{\left|V_{n}\right|^{2}}\right)+Q_{D S T A T}
\end{aligned}
$$

where, $X_{n, n+1}$ is the reactance of the line while $R_{n, n+1}$ denotes its resistance of the line, respectively. $Q_{n}$ and $P_{n}$ denote the flows of active and reactive capabilities, respectively. Real power losses are formulated as follows:

$$
P_{l o s s(n, n+1)}=R_{n, n+1}\left(\frac{P_{n}^{2}+j Q_{n}^{2}}{\left|V_{n}\right|^{2}}\right)
$$


The stability index is given using (4)

$$
V S I_{(n+1)}=\left|V_{n}\right|^{4}-4\left(P_{m n+1} X_{n}-Q_{n+1} R_{n}\right)^{2}-4\left(P_{n+1} X_{n}+Q_{n+1} R_{n}\right)\left|V_{n}\right|^{2}
$$

The voltage deviations of RDN is given using (5)

$$
V D=\sum_{h=1}^{n}\left|V_{n}-1\right|
$$

where, $n$ represents number of buses.

\subsection{Objective function}

The purpose of installation the DSTATCOMs along with PV system is reducing the loss as well as the voltage profile and stability boosting. Therefore, the fitness function is is considered as follows:

$$
k=L_{1} k_{1}+L_{2} k_{2}+L_{3} k_{3}
$$

where, $L_{1}, L_{2}$ and $L_{3}$ denotes the weighting factors which can be formulated using (7):

$$
\left|L_{1}\right|+\left|L_{2}\right|+\left|L_{3}\right|=1
$$

where $k_{1}$ denotes the first fitness function of the power losses of the multi-target function which represents the total reduction of active energy losses and can be found as follows:

$$
k_{1}=\frac{\left(P_{T, \text { loss }}\right)_{P V \& D S T A T C O M}}{\left(P_{T, \text { loss }}\right)_{\text {base }}}
$$

where, $\left(P_{T, \text { loss }}\right)_{\text {PV\&DSTATCOM }}$ is the power losses with optimal integration of PV or DSTATCOM. $\left(P_{T, \text { loss }}\right)_{\text {base }}$ denotes the power loss with at base case. $k_{2}$ represents the of the boosting voltage profile which has been formulated as:

$$
k_{2}=\frac{(V D)_{P V \& D S T A T C O M}}{(V D)_{\text {base }}}
$$

where, $(V D)_{P V \& D S T A T C O M}$ is the $V D$ with optimal integration of PV or DSTATCOM. $(V D)_{\text {base }}$ denotes the $V D$ at base case.

$k_{3}$ is the voltage stability boosting which can be given using (10) as follows:

$$
k_{3}=\frac{1}{\sum_{i=1}^{n b}|\operatorname{VSI}(i)|_{P V \& D S T A T C O M}}
$$

\subsection{The System constraints}

The system limitations are classified as follows:

\subsubsection{Equality constraints}

$$
\begin{aligned}
& P_{S}+\sum_{i=1}^{n p} P_{P V}=\sum_{h=1}^{n} P_{D}(h)+\sum_{j=1}^{n l} P_{\text {loss }}(j) \\
& Q_{S}+\sum_{i=1}^{n c} Q_{\text {DSTATcom }}(i)=\sum_{h=1}^{n} Q_{D}(h)+\sum_{j=1}^{n l} Q_{\text {loss }}(j)
\end{aligned}
$$


where, $P_{S}$ is the active injected power at slack bus while $Q_{S}$ the reactive power. $P_{D}$ the active demand while $Q_{D}$ is the reactive load, respectively. $n c$ denotes number of DSTATCOMs. $n p$ represents number of PV units.

\subsubsection{Inequality constraints}

$$
\begin{aligned}
& V_{\text {min }} \leq V_{i} \leq V_{\text {max }} \\
& \sum_{i=1}^{n c} Q_{D S T A T C O M}(i) \leq \sum_{i=1}^{n} Q_{D}(i) \\
& \sum_{i=1}^{n p} P_{P V}(i) \leq \sum_{i=1}^{n} P_{D}(i) \\
& I_{n} \leq I_{\text {max }, n}, \quad n=1,2,3 \ldots, N_{b}
\end{aligned}
$$

where, $V_{\min }$ and $V_{\max }$ are the lower limit and upper voltage limit. $Q_{\text {DSTATCoM }}$ is injected with reactive power by DSATACOM. $P_{P V}$ is injected with reactive power by PV units.

\subsection{Slime Mould Algorithm (SMA)}

This algorithm simulates a method for finding multiple heads in a vesarium that inhabits cold and wet places. In this technique, weights approach to negative and positive feedbacks created by sticky mold during the foraging process. During this stage, the slurry can determine the best food-gathering path in a superior way. The organic matter in the slime mold searches for food. Then it encircles it and releases enzymes for its consumption. In the migration stage, the anterior end expands into a fan shape along with a venous network that allows the cytoplasm to slide inward. An intravenous network consists of using multiple food sources simultaneously to form to connect them. In this mechanism, a reproductive wave is formed when a vein approaches a food source. The mathematical representation of MSA is formulated as follows:

\subsubsection{Approach food}

The shrinkage mode of a slime mold can be represented as follows [34]:

$$
X(t+1)=\left\{\begin{array}{lr}
X_{b}(t)+v b \cdot\left(W \cdot X_{A}(t)-X_{B}(t)\right) & r<p \\
v_{c} \cdot X(t), & r \geq p
\end{array}\right.
$$

where $v_{b}$ denotes a parameter with a range of $[-a, a], v_{c}$ is reduced from one to zero. $t$ denotes the current iteration, $X_{b}$ is the location of slime mould with the highest odor concentration currently assigned, $X$ denotes the location of slime mould, $X_{A}$ and $X_{B}$ are two individuals randomly selected from the swarm, $W$ represents the weight of slime mould. The $p$ is a parameter which can be obtained as follows:

$$
p=\tanh |S(i)-D F|
$$

where $i \in 1,2, \ldots, n, S(i)$ denotes the objective function of $\vec{X}, D F$ is the best objective function obtained in all iterations. $v b$ is calculated as follows:

$$
\begin{aligned}
& v_{b}=[-a, a] \\
& a=\operatorname{arctanh}\left(-\left(\frac{t}{\max \_t_{-}}\right)+1\right)
\end{aligned}
$$

The $W$ is depicted using (21) as follows:

$$
W=\left\{\begin{array}{c}
1+r \cdot \log \left(\frac{b F-S(i)}{b F-w F}+1\right), \text { condition } \\
1-r \cdot \log \left(\frac{b F-S(i)}{b F-w F}+1\right), \text { others }
\end{array}\right.
$$




$$
\text { SmellIndex }=\operatorname{sort}(S)
$$

where condition indicates that $S(i)$ classifies the first half of the population, $r$ represents a random parameter in rang $[0,1], b F$ represents the optimal objective function, $w F$ represents the worst objective function.

\subsection{Wrap food}

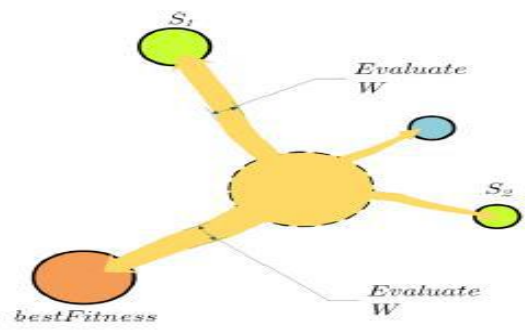

Fig. 2. Evaluation of fitness.

The case simulates a slime mold to control research patterns related to food quality. If the food concentration is contained, the weight near the area is greater; when the concentration of food is low, the weight of the area will decrease, and thus it will turn to explore other areas. Fig. 2 illustrates the process of evaluating the fit values of a slime mold. Based on the principle above, the mathematical formula for updating a slime mold site is as follows:

$$
X^{*}=\left\{\begin{array}{l}
\text { rand } \cdot(U B-L B)+L B, \text { rand }<z \\
X_{b}(t)+v_{b} \cdot\left(W \cdot X_{A}(t)-X_{B}(t)\right), r<p \\
v c \cdot X(t), r \geq p
\end{array}\right.
$$

where $L B$ is the lower bound of control variable while $U B$ is its maximum, rand and $r$ are random variables which equal to $1 \geq r$, rand $\geq 0$.

\subsection{Grabble food}

The $v_{b}$ varied randomly within rang $[-a, a]$ and gradually go to zero with as increasing the iterations number. The value of $v c$ varied between $[-1,1]$ and reached to zero eventually.

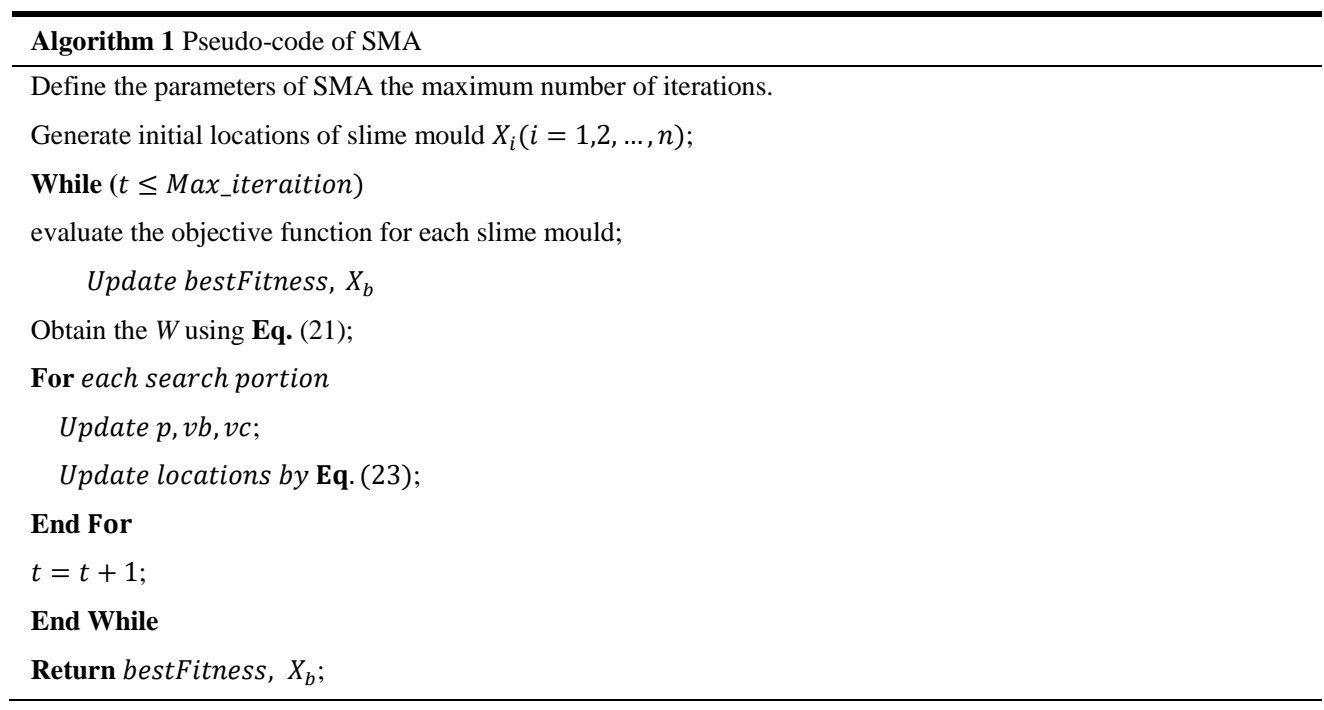




\section{Simulation Results}

TABLE.1. PARAMETERS OF SYSTEM

\begin{tabular}{cc}
\hline Specification & Value \\
\hline Max iteration & 100 \\
slime moulds & 25 \\
Voltage bounds & $0.90 \leq V_{i} \leq 1.05 \mathrm{p} . \mathrm{u}$ \\
PV sizing bounds & $0 \leq P_{D G} \leq 23 \mathrm{MW}$ \\
DSTATCOM sizing bounds & $0 \leq Q_{\text {STATCOM }} \leq 14 \mathrm{MVar}$ \\
\hline
\end{tabular}

The numerical results are shown in this part where the positioning and evaluation of PV modules and DSTATCOMs are made using SMA distribution in the East Delta Network (EDN) as part of the Unified Egyptian Network (UEN) network. System constraints and SMA parameters are tabulated in Table 1. EDN system data are provided in [34].

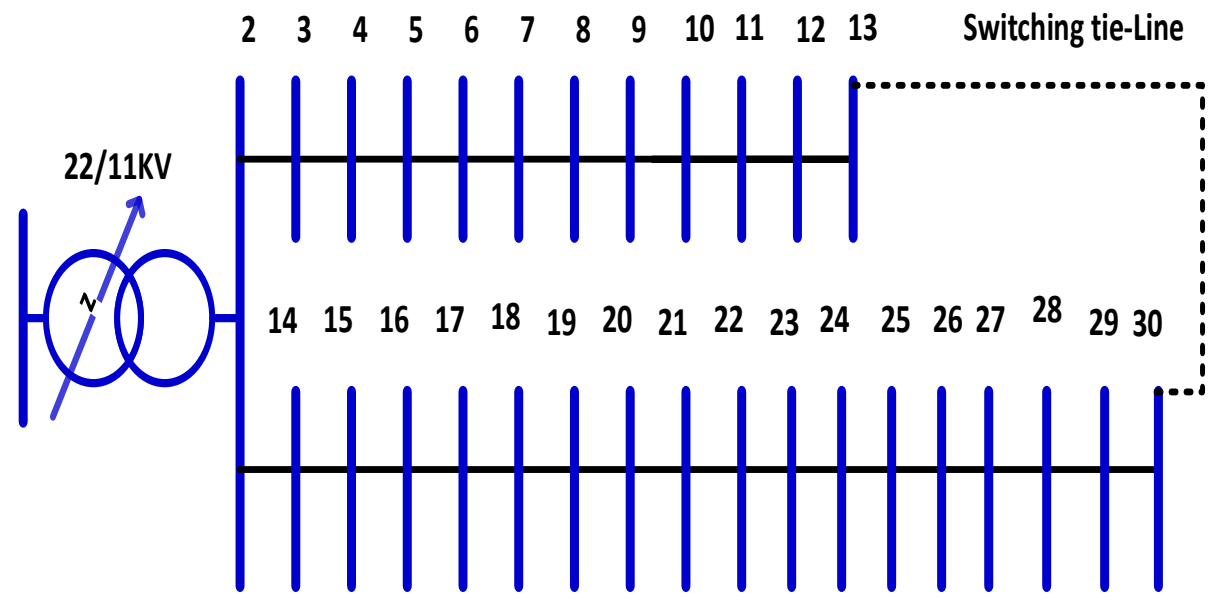

Fig. 3. Single line diagram of the (EDN)

Fig. 3. Shows the single EDN schematic diagram. The system load demand is $11 \mathrm{kV}$, the system load is $22,441,259 \mathrm{~kW}+\mathrm{j} 14,162,265 \mathrm{kV}$. The minimum voltage value is $0.94626 \mathrm{p}$.u on bus 30 . The total active loss is $805.733 \mathrm{~kW}$. Three case studies were presented including optimized assignment of DSTATCOMs only, optimized allocation of PV only modules and optimized assignment of DSTATCOM along with PV modules. All simulations are listed in Table 2. Judging from Table 2, it is clear that the active power loss has decreased from 805.733 (base case) to $790.5877 \mathrm{~kW}, 339.7244 \mathrm{~kW}$, and $123.0589 \mathrm{~kW}$ with optimal integration of DSTATCOMs only, and installation of PV modules only and PV modules were installed with DSTATCOMs, respectively. Improved voltage profile from 1.0669 p.u. (Base case) to 0.6698 (using DSTATCOM only) p.u, 0.1496 p.u (with PV only) and 0.0652 p.u (with PV and DSTATCOM). The voltage stability indicator has also been improved. Improved voltage profile from 24.9867 p.u. (Base case) to 26.4203 (with DSTATCOM only) 28.4080 pu (with PV only) and 28.8183 p.u (with PV and DSTATCOM. From the results obtained, the system is greatly improved with optimized allocation of PV and DSTATCOM simultaneously) Compared with the optimized assignment of PV only or DSTATCOM. The voltage profile has been depicted in Fig 4; it can be evident that PV modulation with DSTATCOM can enhance the voltage profile greatly. Referring to Table 2, voltage stability also enhanced PV modulation with DSTATCOM. Fig. 5 that the proposed algorithm has excellent and stable convergence characteristics as no fluctuation appears. 
TABLE.2. SiMULATION RESULTS WITH OR WITHOUT PV UNITS AND DSTATCOM.

\begin{tabular}{|c|c|c|c|c|}
\hline Values & Basic & DSTATCOM & PV & PV \& DSTATCOM \\
\hline \multirow{2}{*}{ Optimal PV Location (Size kW) } & \multirow{2}{*}{ - } & \multirow{2}{*}{-} & $11(4894.5)$ & $20(11927)$ \\
\hline & & & $(14627) 19$ & $7(6676)$ \\
\hline \multirow{3}{*}{$\begin{array}{c}\text { Optimal DSTATCOM Location } \\
\text { (Size kVar) }\end{array}$} & \multirow{3}{*}{-} & \multirow{3}{*}{$\begin{array}{c}19(13924) \\
6(8327)\end{array}$} & \multirow{3}{*}{-} & \multirow{3}{*}{$\begin{array}{l}16(9878.9) \\
12(1400)\end{array}$} \\
\hline & & & & \\
\hline & & & & \\
\hline Total $\mathrm{P}_{\mathrm{PV}}(\mathrm{kW})$ & - & - & $19,521.5$ & 18,603 \\
\hline $\begin{array}{c}\text { Total } Q_{\text {DSTATCOM }} \\
\text { (KVar) }\end{array}$ & - & 22251 & - & $11,278.9$ \\
\hline$V_{\min }(p . u) @$ bus no. & $0.94626 @$ bus 30 & $0.96422 @$ bus 30 & $0.99038 @$ bus 30 & $0.99437 @$ bus 30 \\
\hline Total $\mathrm{P}_{\text {losses }}(\mathrm{kW})$ & 805.733 & 790.5877 & 339.7244 & 123.0589 \\
\hline Total $Q_{\text {loss }}(\mathrm{kVar})$ & 61.184 & 347.708 & 143.005 & 46.890 \\
\hline$V D(p . u)$ & 1.0669 & 0.6698 & 0.1496 & 0.0652 \\
\hline$\sum V S I$ & 24.9867 & 26.4203 & 28.4080 & 28.8183 \\
\hline
\end{tabular}

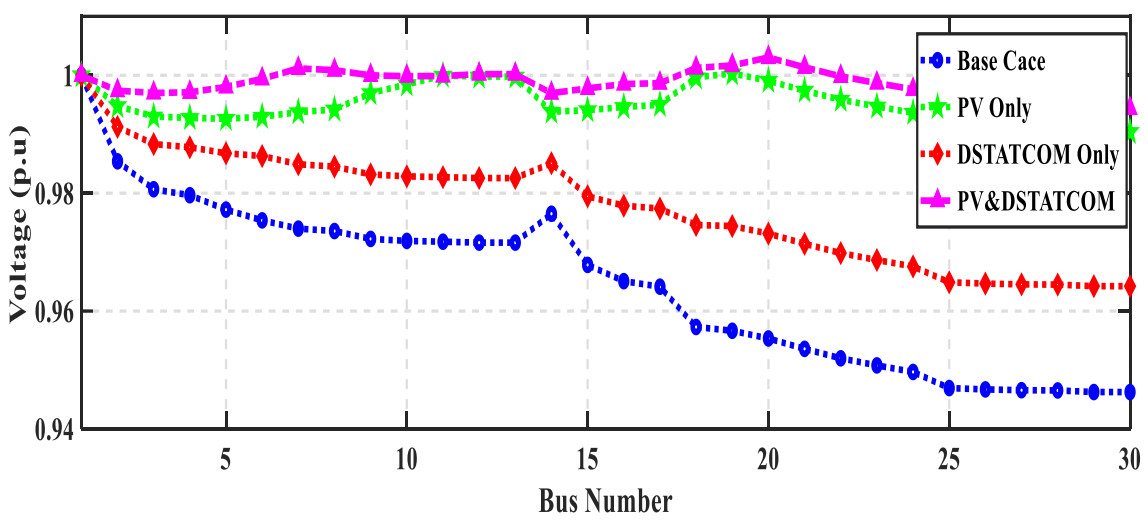

Fig .4. The system voltage profile.

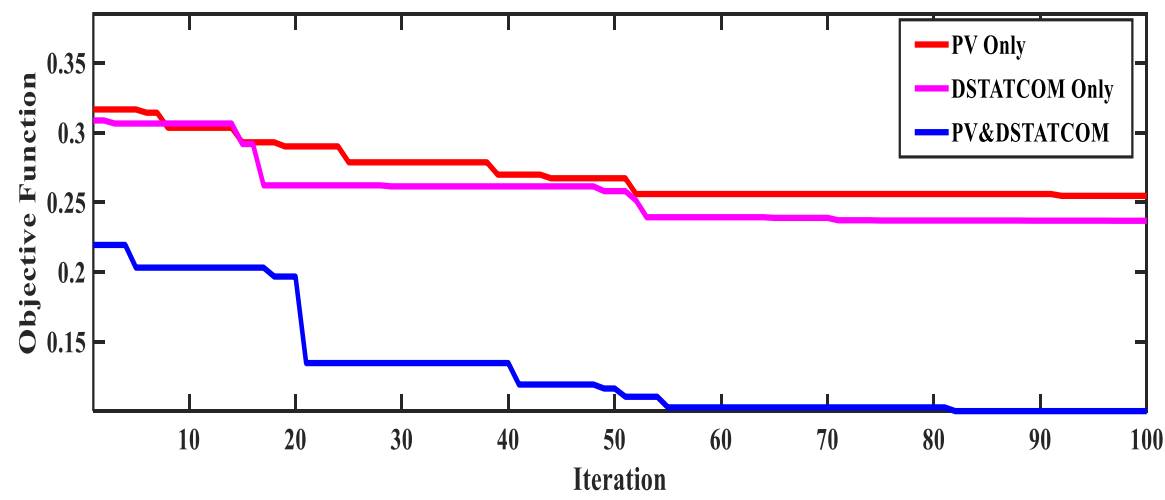

Fig .5. The convergence characteristic of the SMA for power losses minimization. 


\section{Conclusion}

This paper has presented an efficient method for optimal sizes and locations of DSTATCOMs and PV system in a true distribution network (East Delta Network). A new optimization technique called Slime Mold Algorithm (SMA) has been applied to set the best locations and sizes of PV and DSTATCOMs to reduce power loss, voltage stability, and improve the voltage profile. Outcomes confirmed that SMA is an effective algorithm to solve the presented problem. In addition, the optimized allocation of PV modules and DSTATCOM can greatly improve performance by reducing active system losses, improving system voltage profile and system stability.

\section{References}

[1] F. Peng, H. Su, P. Li, G. Song, and J. Zhao, "An interactive operation strategy for microgrid cooperated with distribution system based on demand response," in 2015 5th International Conference on Electric Utility Deregulation and Restructuring and Power Technologies (DRPT), 2015, pp. 156-160.

[2] A. J. G. Mena and J. A. M. García, "An efficient approach for the siting and sizing problem of distributed generation," International journal of electrical power \& energy systems, vol. 69, pp. 167-172, 2015.

[3] M. Gomez-Gonzalez, A. López, and F. Jurado, "Optimization of distributed generation systems using a new discrete PSO and OPF," Electric Power Systems Research, vol. 84, pp. 174-180, 2012.

[4] F. Shariatzadeh, N. Kumar, and A. K. Srivastava, "Intelligent control algorithms for optimal reconfiguration of microgrid distribution system," in 2015 IEEE Industry Applications Society Annual Meeting, 2015, pp. 1-8.

[5] S. A. Arefifar, Y. A.-R. I. Mohamed, and T. El-Fouly, "Optimized multiple microgrid-based clustering of active distribution systems considering communication and control requirements," IEEE transactions on industrial electronics, vol. 62, pp. 711-723, 2014.

[6] H. Ng, M. Salama, and A. Chikhani, "Capacitor allocation by approximate reasoning: fuzzy capacitor placement," IEEE transactions on power delivery, vol. 15, pp. 393-398, 2000.

[7] S. Sharma and S. Ghosh, "FIS and hybrid ABC-PSO based optimal capacitor placement and sizing for radial distribution networks," Journal of Ambient Intelligence and Humanized Computing, vol. 11, pp. 901-916, 2020.

[8] M. Ebeed, S. Kamel, S. H. A. Aleem, and A. Y. Abdelaziz, "Optimal allocation of compensators," in Electric distribution network planning, ed: Springer, 2018, pp. 321-353.

[9] F. Z. Peng, "Flexible AC transmission systems (FACTS) and resilient AC distribution systems (RACDS) in smart grid," Proceedings of the IEEE, vol. 105, pp. 2099-2115, 2017.

[10] N. G. Hingorani, "FACTS technology-state of the art, current challenges and the future prospects," in IEEE Power Engineering Society General Meeting, 2007.

[11] S. Kamel, F. Jurado, and D. Vera, "A simple implementation of power mismatch STATCOM model into current injection Newton-Raphson power-flow method," Electrical Engineering, vol. 96, pp. 135-144, 2014.

[12] B. V. Kumar, "Optimal Location of Upfc to Improve Power System Voltage Stability Using Artificial Bee Colony Algorithm," American Journal of Electrical Power and Energy Systems, vol. 8, p. 42, 2019.

[13] N. A. Gajjar and T. N. Zaveri, "A Review of DSTATCOM Used in Solar Photovoltaic System," in 2018 International Conference and Utility Exhibition on Green Energy for Sustainable Development (ICUE), 2018, pp. 1-7.

[14] O. P. Mahela and A. G. Shaik, "A review of distribution static compensator," Renewable and Sustainable Energy Reviews, vol. 50, pp. 531-546, 2015.

[15] R. Majumder, "Reactive power compensation in single-phase operation of microgrid," IEEE transactions on industrial electronics, vol. 60, pp. 1403-1416, 2012.

[16] G. Mokhtari, G. Nourbakhsh, F. Zare, and A. Ghosh, "A new distributed control strategy to coordinate multiple dstatcoms in LV network," in 2013 4th IEEE International Symposium on Power Electronics for Distributed Generation Systems (PEDG), 2013, pp. 1-5.

[17] F. Shahnia, R. P. Chandrasena, A. Ghosh, and S. Rajakaruna, "Application of DSTATCOM for surplus power circulation in MV and LV distribution networks with single-phase distributed energy resources," Electric Power Systems Research, vol. 117, pp. 104-114, 2014.

[18] L. Zhang, "Modeling and control of VSC-HVDC links connected to weak AC systems," KTH, 2010.

[19] S. Ananthavel, S. Padmanaban, S. Shanmugham, F. Blaabjerg, A. H. Ertas, and V. Fedak, "Analysis of enhancement in available power transfer capacity by STATCOM integrated SMES by numerical simulation studies," Engineering Science and Technology, an International Journal, vol. 19, pp. 671-675, 2016.

[20] N. Jenkins and G. Strbac, "Effects of small embedded generation on power quality," in IEE Colloquium on Issues in Power Quality, 1995, pp. 6/1-6/4.

[21] R. Saint and N. Friedman, "The application guide for distributed generation interconnection-the NRECA guide to IEEE 1547," in 2002 Rural Electric Power Conference. Papers Presented at the 46th Annual Conference (Cat. No. 02CH37360), 2002, pp. D2-1. 
[22] E. S. Oda, A. M. Abd El Hamed, A. Ali, A. A. Elbaset, M. Abd El Sattar, and M. Ebeed, "Stochastic Optimal Planning of Distribution System Considering Integrated Photovoltaic-Based DG and DSTATCOM Under Uncertainties of Loads and Solar Irradiance," IEEE Access, vol. 9, pp. 26541-26555, 2021.

[23] H. M. Sultan, A. S. Menesy, S. Kamel, A. Korashy, S. Almohaimeed, and M. Abdel-Akher, "An improved artificial ecosystem optimization algorithm for optimal configuration of a hybrid PV/WT/FC energy system," Alexandria Engineering Journal, vol. 60, pp. 1001-1025, 2021.

[24] D. Ahmed, M. Ebeed, A. Ali, A. S. Alghamdi, and S. Kamel, "Multi-Objective Energy Management of a Micro-Grid Considering Stochastic Nature of Load and Renewable Energy Resources," Electronics, vol. 10, p. 403, 2021.

[25] V. Janamala, "A new meta-heuristic pathfinder algorithm for solving optimal allocation of solar photovoltaic system in multi-lateral distribution system for improving resilience," SN Applied Sciences, vol. 3, pp. 1-17, 2021.

[26] S. A. Taher and S. A. Afsari, "Optimal location and sizing of DSTATCOM in distribution systems by immune algorithm," International Journal of Electrical Power \& Energy Systems, vol. 60, pp. 34-44, 2014.

[27] S. Devi and M. Geethanjali, "Optimal location and sizing determination of Distributed Generation and DSTATCOM using Particle Swarm Optimization algorithm," International Journal of Electrical Power \& Energy Systems, vol. 62, pp. 562-570, 2014.

[28] S. Mishra and P. K. Ray, "Power quality improvement using photovoltaic fed DSTATCOM based on JAYA optimization," IEEE Transactions on Sustainable Energy, vol. 7, pp. 1672-1680, 2016.

[29] T. Yuvaraj, K. Ravi, and K. Devabalaji, "DSTATCOM allocation in distribution networks considering load variations using bat algorithm," Ain Shams Engineering Journal, vol. 8, pp. 391-403, 2017.

[30] T. Yuvaraj, K. Devabalaji, and K. Ravi, "Optimal placement and sizing of DSTATCOM using harmony search algorithm," Energy Procedia, vol. 79, pp. 759-765, 2015.

[31] A. Ramadan, M. Ebeed, and S. Kamel, "Performance assessment of a realistic egyptian distribution network including PV penetration with DSTATCOM," in 2019 International Conference on Innovative Trends in Computer Engineering (ITCE), 2019, pp. 426-431.

[32] M. A. E. S. Mohamed, A. A. E. Mohammed, A. M. Abd Elhamed, and M. E. Hessean, "Optimal allocation of photovoltaic based and DSTATCOM in a distribution network under multi load levels," European Journal of Engineering and Technology Research, vol. 4, pp. 114-119, 2019.

[33] S. Li, H. Chen, M. Wang, A. A. Heidari, and S. Mirjalili, "Slime mould algorithm: A new method for stochastic optimization," Future Generation Computer Systems, vol. 111, pp. 300-323, 2020.

[34] D. T. Abdul-hamied, A. M. Shaheen, W. A. Salem, W. I. Gabr, and R. A. El-sehiemy, "Equilibrium optimizer based multi dimensions operation of hybrid AC/DC grids," Alexandria Engineering Journal, 2020. 